\title{
Phenolic compounds in strawberry (Fragaria $x$ ananassa Duch.) microshoots
}

\author{
Olga Kotsupiy $^{1 *}$, Evgeniya Karpova ${ }^{1}$, and Elena Ambros ${ }^{1}$ \\ ${ }^{1}$ Central Siberian Botanical Garden, SB RAS, 630090 Zolotodolinskaya str., 101, Novosibirsk, Russia
}

\begin{abstract}
The HPLC method was used to study the composition and contents of phenolic compounds in the microshoots of Fragaria $\times$ ananassa Duch. plantlets during the multiplication and in vitro rooting stages in comparison with field-grown plants. Phenolic compounds of the microshoots are predominantly represented by ellagic acid and its derivatives, as well as hydroxycinnamic and hydroxybenzoic acids, in contrast to the leaves of field-grown plants with a high level of flavonoids. The increased contents of individual hydroxybenzoic and hydroxycinnamic acids in the microshoots compared with the leaves of field-grown strawberry plants indicates a possible role of phenolic acids in plant adaptation under in vitro conditions.
\end{abstract}

\section{Introduction}

Cultivated strawberry (Fragaria $\times$ ananassa Duch.) is one of the most popular berry fruits in many countries of the world. However, long-term cultivation of this species leads to the accumulation of pathogens that inhibits growth and reduces crop productivity. Micropropagation techniques are a suitable alternative for pathogen-free rapid propagation of strawberry cultivars. Initial stages of micropropagation are regeneration on a medium supplemented with a high concentration of cytokinins and elongation on a medium without plant growth regulators [1]. In vitro rooting of microshoots is the key stage of micropropagation that determines the survival of plantlets in ex vitro conditions. The protocol provides the high rates of shoot multiplication and the formation of plantlets with optimal physiological status for ex vitro acclimatization. This is evidenced by the high contents of photosynthetic pigments in the leaves of strawberry plantlets (chlorophylls $a$ and $b$, and carotenoids), and their ratios (chlorophyll $a / b$, chlorophyll $(a+b) /$ carotenoids) [2]. On the other hand, in vitro conditions are stressful for plants (high relative humidity, low gas exchange with the external environment, the presence of exogenous carbohydrates and growth regulators, and low $\mathrm{CO}_{2}$ absorption rate). These in vitro factors interfere the successful acclimatization of plants under non-sterile conditions. In this regard, it is of interest to expand the spectrum of biochemical indicators of the adaptive status of strawberry plants with secondary metabolites, including phenolic compounds, which can often serve as indicators of stress and adaptation [3]. The composition of phenolic compounds of $F . \times$ ananassa leaves was studied fragmentarily. Ellagic and gallic acids,

\footnotetext{
*Corresponding author: olnevaster@gmail.com
} 
their derivatives; vanillic, p-hydroxybenzoic, caffeic, chlorogenic acids, as well as flavonols: quercetin, kempferol, myricetin and their glycosides, including rutin, were found in strawberry leaves. Meanwhile, ellagic acid derivatives can constitute up to $50 \%$ of phenolic compounds $[4,5]$. Flavonoid concentrations also reached significant values (up to $30 \%$ of total phenolic content) [6,7]. A comparison of phenolic compositions in $F$. $\times$ ananassa plants cultivated at the different conditions will help identify the major compounds for the adaptations to each type of environments, including in vitro conditions.

The objective of the present investigation was to study the composition and contents of phenolic compounds in the microshoots of $F$. $\times$ ananassa plantlets during the multiplication and in vitro rooting in comparison with field-grown plants.

\section{Materials and Methods}

\subsection{Plant material and culture conditions}

The microshoots $(1.0 \mathrm{~cm})$ with two leaves and apical bud of strawberry cultivar, 'Solnechnaya polyanka', were used as explants for micropropagation. Shoot multiplication was carried out by activation of axillary meristems of growing microshoots on GamborgEveleg's $\left(\mathrm{B}_{5}\right)$ [8] nutrient medium supplemented with $0.75 \mathrm{mg} \mathrm{L}^{-1}$ 6-benzylaminopurine (plant cell culture tested, BioReagent, SigmaAldrich ${ }^{\circledR}$ ). The $\mathrm{pH}$ of the medium was adjusted to 5.5 with $1 \mathrm{M} \mathrm{NaOH}$ or $\mathrm{HCl}$ and was solidified by using Bacto ${ }^{\circledR}$ agar (PanReac ${ }^{\circledR}$, Barcelona, Spain) at $6.0 \mathrm{~g} \mathrm{~L}^{-1}$ prior to autoclaving $\left(121^{\circ} \mathrm{C}, 20 \mathrm{~min}, 1.05 \mathrm{~kg}\right.$ $\mathrm{cm}^{-2}$ ). In vitro rooting was performed on a hormone-free medium of the same composition. The cultures were maintained in culture jars $(15 \mathrm{~mL}$ medium per vessel $)$ at $23 \pm 2^{\circ} \mathrm{C}$ with a 16-h photoperiod with $40 \mu \mathrm{mol} \mathrm{m} \mathrm{m}^{-2} \mathrm{~s}^{-1}$ of light intensity provided by cool white fluorescent lamps (Philips, Pila, Poland). The duration of the micropropagation stages was 8 weeks. Field-grown plant leaves were collected from the experimental plot of the biotechnology laboratory of the Central Siberian Botanical Garden of the SB RAS (Novosibirsk) (geographical coordinates $54^{\circ} 49^{\prime} 9.87^{\prime \prime} \mathrm{N}$ and $83^{\circ} 6^{\prime} 6.95^{\prime \prime} \mathrm{E}$ ) in 2019 . The average samples of the strawberry microshoots and the leaves of generative field-grown plants in the flowering phase were dried in air in the shade to a moisture content of $10 \%$ and taken for analysis of phenolic compounds.

\subsection{Extraction, sample preparation and HPLC conditions}

For phenolic compounds extraction, precisely weighted powdered air-dried plant material was exhaustively extracted with an ethanol:water mixture $(70: 30, \mathrm{v} / \mathrm{v})$ in a water bath at $60-70^{\circ} \mathrm{C}$. For acid hydrolysis, $0.5 \mathrm{ml}$ of water-ethanol extraction was added to $0.5 \mathrm{ml}$ of $\mathrm{HCl}(2 \mathrm{~N})$ and heated in a boiling water bath for 2 hours. The components were analyzed using the HPLC system consisted of an Agilent 1200 with a diode array detector and the ChemStation software (Agilent Technologies, USA) for data processing. The chromatographic separation was conducted at $25^{\circ} \mathrm{C}$ on a Zorbax SB-C18 Column $(4.6 \times$ $150 \mathrm{~mm}, 5 \mu \mathrm{m}$ i.d.) in the gradient elution mode. The extracts and the hydrolysates were separated with gradient 1 and gradient 2, respectively. In gradient 1 , methanol concentration in an aqueous solution of orthophosphoric acid $(0.1 \%)$ changed from 22 to $70 \%$ within 30 minutes, then to $100 \%$ by 32 minute, and in gradient 2 , the methanol concentration increased from 50 to $100 \%$ within 17 minutes. The flow rate was set to 1 $\mathrm{mL} \cdot \mathrm{min}^{-1}$. The sample injection volume was $10 \mu \mathrm{L}$, and the absorbance was measured at $210,255,270,290,325,340,360$, and $370 \mathrm{~nm}$. Qualitative analysis of phenolic compounds was performed based on the peaks of reference standards of gallic, chlorogenic, cinnamic, 
caffeic, ferulic, p-coumaric, ellagic acids, quercetin, and kaempferol (Sigma-Aldrich, St. Louis, MO, USA). Quantification of ellagic acid, its derivatives and phenolic acids; flavonoid aglycons, and flavonoid glycosides was carried out according to the calibration curve for ellagic acid, quercetin and rutin, respectively, in the concentration range of 10$100 \mu \mathrm{g} \cdot \mathrm{ml}^{-1}$. The concentrations of the phenolic compounds were expressed in mg per 100 $\mathrm{g}$ of absolutely dry weight (DW). Each sample was analyzed in three replicates.

All the data were processed in the Statistica 10.0 software (Statsoft Inc., Tulsa, OK, USA), were reported as mean \pm standard error (SE) of three replicates, and were compared using ANOVA followed by Duncan's multiple range test. Differences between the means were considered statistically significant at the $5 \%$ level $(\mathrm{p}<0.05)$.

\section{Results}

In phenolic composition of $F . \times$ ananassa microshoots, citric, gallic, caffeic, ferulic, pcoumaric, and ellagic acids were identified, as well as unidentified compounds with absorption maxima typical for hydroxybenzoic, hydroxycinnamic acids, and ellagic acid derivatives (Fig.). These compounds, along with flavonoid components with absorption maxima in the range of $350-360 \mathrm{~nm}$, were found in the leaves of field-grown plants. The composition of hydrolysates indicates that they are quercetin and kaempferol glycosides.
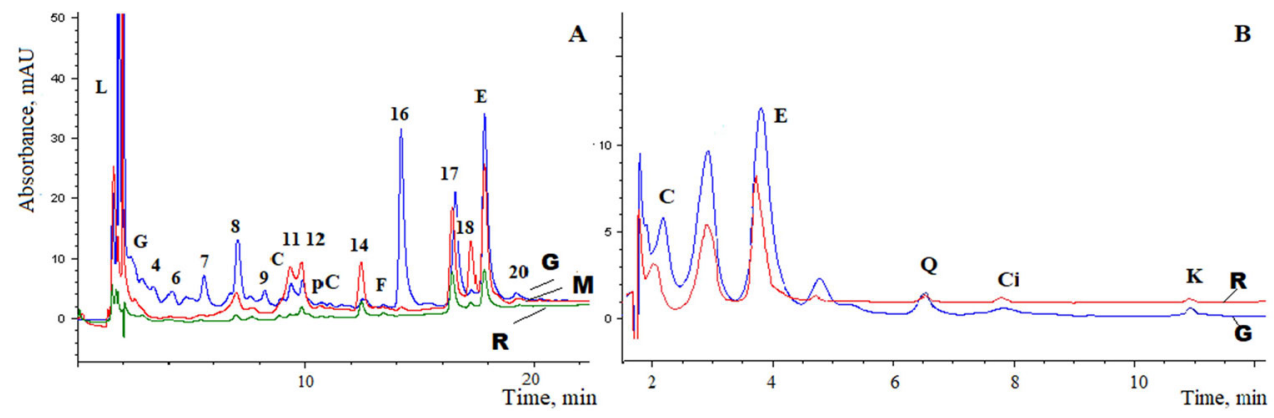

Fig. Representative section of the HPLC chromatograms of the extracts (A) and the hydrolisates (B) of the above-ground parts of 'Solnechnaya Polyanka' plants. M multiplication, $\mathrm{R}$ in vitro rooting, $\mathrm{G}$ field-grown plants, $\mathrm{L}$ citric acid, $\mathrm{G}$ gallic acid, $\mathrm{C}$ caffeic acid, $\mathrm{pC}$ p-coumaric acid, $\mathrm{F}$ ferulic acid, $\mathrm{E}$ ellagic acid, Q quercetin, Ci cinnamic acid, K kaempferol

The composition of the main constituents of the leaves of field-grown plants was more extensive than that of microshoots for account flavonoids and some hydroxybenzoic acids (Fig., table). The field-grown plants exceeded the in vitro plants during multiplication and in vitro rooting in the following individual phenolic compounds: gallic acid by 1.6 and 2.2 times, respectively, hydroxycinnamic acid $\mathbf{4}$ by 2.5 and 3 times, citric acid by 1.6 and 3.2 times, hydroxybenzoic acids 6-8 by 1.4-7.5 times, and ellagic acid by 1.4 times (Table). However, the concentrations of certain compounds in the microshoots were higher than in the leaves of field-grown plants, and these compounds were different during multiplication and in vitro rooting. Within multiplication, these compounds included hydroxybenzoic acids 11 and 12, and ellagic acid derivatives, and within in vitro rooting they contained caffeic, p-coumaric and ferulic acids. The concentration of compound $\mathbf{1 4}$ increased during both stages of micropropagation.

In the microshoots, quercetin and kaempferol derivatives were detected in trace amounts. The quercetin concentrations in the hydrolysates of the leaves of field-grown plants were 10 times higher, compared to the hydrolysates of the in vitro plantlets. Thus, the higher total phenolic content in the leaves of field-grown plants compared to in vitro 
plantlets is due to a more complete composition of phenolic compounds, including flavonoids and hydroxybenzoic acids not available in in vitro plantlets.

Table. Spectral characteristics and concentrations (mg per $100 \mathrm{~g} \mathrm{DW})$ of major phenolic compounds in the leaves of $F$. $\times$ ananassa in conditions of field $(\mathrm{G})$ and in vitro culture during multiplication $(\mathrm{M})$ and in vitro rooting $(\mathrm{R})$

\begin{tabular}{|c|c|c|c|c|c|c|}
\hline Peak & $\mathrm{t}_{\mathrm{r}}, \min$ & $\lambda_{\max }, \mathrm{nm}$ & Compound & $\mathrm{G}$ & $\mathrm{M}$ & $\mathrm{R}$ \\
\hline $\mathrm{L}$ & 1.8 & 220 & Citric acid & $326.5 \pm 18.3^{\mathrm{a}}$ & $210.8 \pm 17.2^{\mathrm{b}}$ & $102.6 \pm 5.5^{\mathrm{c}}$ \\
\hline 2 & 2.0 & 220,250 & Hb & $268.0 \pm 11.1^{\mathrm{a}}$ & $278.8 \pm 13.4^{\mathrm{a}}$ & $87.8 \pm 4.1^{\mathrm{b}}$ \\
\hline $\mathrm{G}$ & 2.4 & 216,272 & Gallic acid & $165.3 \pm 8.7^{\mathrm{a}}$ & $102.7 \pm 3.1^{\mathrm{b}}$ & $74.5 \pm 3.7^{\mathrm{c}}$ \\
\hline 4 & 2.9 & 327 & Hc & $137.8 \pm 9.5^{\mathrm{a}}$ & $46.3 \pm 3.4^{\mathrm{c}}$ & $54.4 \pm 2.4^{\mathrm{b}}$ \\
\hline 5 & 3.3 & 220,270 & Hb & $102.6 \pm 5.1$ & n. d. & n. d. \\
\hline 6 & 4.0 & 270 & Hb & $101.3 \pm 6.4^{\mathrm{a}}$ & $40.3 \pm 2.9^{\mathrm{b}}$ & $13.5 \pm 0.9^{\mathrm{c}}$ \\
\hline 7 & 5.5 & 220,270 & Hb & $180.5 \pm 9.3^{\mathrm{a}}$ & $52.7 \pm 3.9^{\mathrm{b}}$ & $38.5 \pm 2.1^{\mathrm{c}}$ \\
\hline 8 & 7.0 & 220,270 & Hb & $181.2 \pm 8.7^{\mathrm{a}}$ & $129.9 \pm 8.9^{\mathrm{b}}$ & $60.7 \pm 5.6^{\mathrm{c}}$ \\
\hline 9 & 8.2 & 220,270 & Hb & $125.4 \pm 10.3$ & $\mathrm{n} . \mathrm{d}$. & n. d. \\
\hline $\mathrm{C}$ & 8.6 & 218, & Caffeic acid & $56.2 \pm 3.8^{\mathrm{b}}$ & $44.0 \pm 3.4^{\mathrm{c}}$ & $73.1 \pm 2.7^{\mathrm{a}}$ \\
\hline 11 & 9.4 & 250,324 & Hb & $66.1 \pm 4.3^{\mathrm{b}}$ & $135.3 \pm 9.6^{\mathrm{a}}$ & $25.2 \pm 1.6^{\mathrm{c}}$ \\
\hline 12 & 9.7 & 220,270 & Hb & $86.8 \pm 4.2^{\mathrm{b}}$ & $156.3 \pm 11.5^{\mathrm{a}}$ & $78.7 \pm 2.4^{\mathrm{c}}$ \\
\hline $\mathrm{pC}$ & 10.8 & 226,310 & P-coumaric acid & $40.6 \pm 1.4^{\mathrm{b}}$ & $30.5 \pm 2.5^{\mathrm{c}}$ & $50.7 \pm 1.3^{\mathrm{a}}$ \\
\hline 14 & 12.3 & 220,270 & Hb & $17.9 \pm 1.1^{\mathrm{b}}$ & $131.9 \pm 10.3^{\mathrm{a}}$ & $123.7 \pm 7.0^{\mathrm{a}}$ \\
\hline $\mathrm{F}$ & 13.3 & 218, & Ferulic acid & $24.1 \pm 1.2^{\mathrm{b}}$ & $20.3 \pm 1.1^{\mathrm{c}}$ & $55.6 \pm 2.0^{\mathrm{a}}$ \\
\hline 16 & 14.1 & 257,357 & Q glycoside & $1148.7 \pm 27.5$ & n. d. & n. d. \\
\hline 17 & 16.3 & 254,360 & E derivative & $269.3 \pm 12.3^{\mathrm{b}}$ & $305.5 \pm 16.4^{\mathrm{a}}$ & $250.7 \pm 3.1^{\mathrm{c}}$ \\
\hline 18 & 17.2 & 254,360 & E derivative & $31.0 \pm 1.4^{\mathrm{c}}$ & $174.2 \pm 5.9^{\mathrm{a}}$ & $44.9 \pm 1.3^{\mathrm{b}}$ \\
\hline $\mathrm{E}$ & 17.7 & 254,367 & Ellagic acid & $438.1 \pm 20.1^{\mathrm{a}}$ & $312.2 \pm 3.4^{\mathrm{b}}$ & $317.4 \pm 16.2^{\mathrm{b}}$ \\
\hline 20 & 19.2 & 265,356 & K glycoside & $108.8 \pm 7.0^{\mathrm{a}}$ & $83.3 \pm 4.4^{\mathrm{b}}$ & $97.5 \pm 3.8^{\mathrm{ab}}$ \\
\hline & $\mathrm{T} 0 \mathrm{tal}$ & & & $3876.2 \pm 28.3^{\mathrm{a}}$ & $2255.0 \pm 19.7^{\mathrm{b}}$ & $1549.5 \pm 57.4^{\mathrm{c}}$ \\
\hline
\end{tabular}

$\mathrm{t}_{\mathrm{r}}$ retention time; $\mathrm{Hb}$ hydroxybenzoic acid; Hc hydroxycinnamic acid; $\mathrm{n}$. d. not detected; Q, E, K, and the peak designations correspond with Figure. Means followed by the same letter are not significantly different according to Duncan's test $(\mathrm{P}<0.05)$.

\section{Discussion}

Phenolic composition of strawberry leaves of cultivar 'Solnechnaya Polyanka' in the South of Western Siberia consists of phenolic acids (gallic, caffeic, p-coumaric, and ferulic acids), ellagic acid and its derivatives, and quercetin and kaempferol glycosides. This composition generally corresponds with that of previously studied samples of other $F \times$ ananassa cultivars [4-6]. Phenolic composition of in vitro plants is similar to that of field-grown plants, and it is characterized by trace amounts of flavonoid glycosides and higher, than in field-grown plants, concentrations of certain phenolic acids. Changes in the composition of 
the major phenolic compounds under different conditions indicate the specifics of the components that provide adaptation. Under open field conditions, the adaptation is achieved by quercetin derivatives and phenolic acids, during in vitro proliferation, it realized by hydroxybenzoic acids and ellagic acid derivatives, and during in vitro rooting, it is implemented with hydroxycinnamic acids. Similar accumulation of quercetin glycosides in field-grown plants and their absence in greenhouse plants was noted previously [3]. The protective effect of hydroxycinnamic acids associated with changes in the activity of antioxidant system enzymes under stress has also been shown $[9,10]$. The concentration of phenolic compounds was shown to be higher in the leaves of wild strawberry plants (Fragaria vesca L.) compared to the leaves of in vitro plantlets [11]. The results revealed higher contents of most phenolic compounds and elevated total phenolic contents in fieldgrown plants compared to in vitro plantlets. Microshoots of plantlets at the in vitro rooting stage were characterized by the minimum phenolic content (1.5 times lower than at the multiplication stage).

\section{Conclusion}

For the first time, the phenolic composition of in vitro plantlets in comparison with fieldgrown plants of $F$. $\times$ ananassa was studied. In vitro conditions contributed to a decrease in the total phenolic content and in the percentage of flavonoids in the sum. The content of individual hydroxybenzoic acids and ellagic acid derivatives in microshoots of plantlets increased during multiplication. In vitro rooting stage was characterized by increased concentrations of the certain hydroxycinnamic acids (caffeic, p-coumaric, and ferulic acids).

The work was financially supported by the draft State assignment of the Central Siberian Botanical Garden of the Siberian Branch of the Russian Academy of Sciences No. AAAA-A17-1170126100515 within the framework of a government contract and with material of CSBG representing USFs (Unique Scientific Facilities) "Collections of living plants indoors and outdoors" USU 440534.

\section{References}

1. E. Ambros, Y. Zaytseva, A .A. Krasnikov, T. Novikova, Plant Life of Asian Russia 28, 73 (2017)

2. E. V. Ambros, O. V. Kotsupy, E. A. Karpova, E. G. Trofimova, Yu. G. Zaytseva, T.I. Novikova, Theoretical and Applied Ecology 4, 54 (2019)

3. E. A. Karpova, T. D. Fershalova, A. A. Petruk, Jornal of Stress Physiology \& Biochemistry, 12, 44 (2016)

4. A. M. D. El-Mesallamy, S. A. M. Hussein, M. El. Gerby, M. H. M. Abd El Azim, Natural products. 9, 251 (2013)

5. A. Kårlund, K. Hanhineva, M. Lehtonen, G.J. McDougall, D. Stewart, R.O. Karjalainen, J. Sci. Food Agric. 97, 2182 (2016)

6. M. Simirgiotis, G. Schmeda-Hirschmann J. Food Compos. Anal. 23, 545 (2010)

7. A. Kårlund, J.-P. Salminen, P. Koskinen, J. R. Ahern, M. Karonen, K. Tiilikkala, R. O. Karjalainen, J. Agric. Food Chem. 62, 4592 (2014)

8. O. L. Gamborg, D. E. Eveleigh, Can. Journal of Biochemistry, 46, 417 (1968)

9. J. Loponen, V. Ossipov, K. Lempa, E. Haukioja, K. Pihlaja, Chemosphere 37, 1445 (1998) 
10. Y. Y. Wan, Y. Zhang, L. Zhang, Z. Q. Zhou, X. Li, Q. Shi, X. J. Wang, J.G. Bai, Acta Physiol. Plant. 37, 1706 (2015)

11. A. B. Yildirim, A. U. Turker, Sci. Hortic. April, 169 (2014) 